\title{
INCIDENCES DES EXTRACTIONS DE MATÉRIAUX ALLUVIONNAIRES ET DE L'AMÉNAGEMENT DES COURS D'EAU SUR L'ECOSYSTÈME AQUATIQUE
}

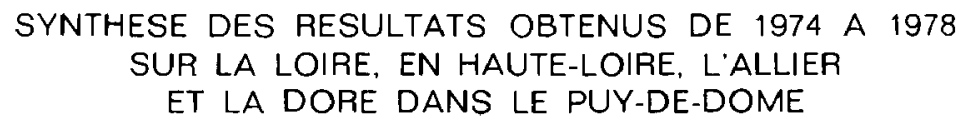

B. BOUCHAUD (1), P. CLAVEL (2), Y. HAMON (3), C. ROMANEIX (3)

\section{AVEC L'AIDE FINANCIERE OU TECHNIQUE}

- du Ministere de l'Environnement et du Cadre de Vie (Direction de la Protection de la Nature)

- du Fonds de Gestion de la Taxe Parafiscale sur les Granulats

- du Conseil Supérieur de la Pêche

-- de I'U.E.R. Sciences, Université Clermont II

(1) Technicien Superieur Agricole \& Protection de la Nature*

(2) Docteur $3^{\circ}$ cycle en Biologie Animale (Hydrobiologie)

(3) Ingénieurs de I'E.S.IT.P.A. 


\section{SOMMAIRE}

\section{CIRCONSTANCES ET OBJET DE L'ETUDE}

RESUME

\section{A. LES MODIFICATIONS DU BIOTOPE}

1 - Morphodynamique du lit.

2 - Physico-chimie de l'eau.

3 - Transmission de l'énergie lumineuse

4 - Sédimentation des matières en suspension.

\section{B. LES MODIFICATIONS DES BIOCENOSES}

1 - Le périphyton.

2 - Les macrophytes.

3 - Les macroinvertébrés benthiques.

4 - Les poissons.

\section{LES MOYENS DE LUTTE CONTRE CE tYPE DE NUISANCES}

1 - Aspect juridique.

2 - Mesures techniques.

a) Les exploitations de granulats.

b) Les aménagements de cours d'eau.

\section{BIBLIOGRAPHIE SOMMAIRE.}

\section{RESUME}

\section{INCIDENCES DES EXTRACTIONS DES MATERIAUX ALLUVIONNAIRES ET DE L'AMENAGEMENT DES COURS D'EAU SUR L'ECOSYSTEME AQUATIQUE}

Les exploitations de granulats (dragages dans le lit mineur, et/ou rejets, mal décantés, d'eau de lavage des sables et graviers extraits) et certains travaux hydrauliques (rectifications, recalibrages), provoquent d'importantes perturbations dans l'écosystème aquatique.

Nous avons essayé, entre 1974 et 1978, de les mettre en évidence sur troıs cours d'eau du MASSIF CENTRAL : la LOIRE, I'ALLIER; la DORE.

LES MODIFICATIONS DU BIOTOPE portent essentiellement sur l'uniformisation et la déstabilisation du lit, l'augmentation du taux de matières en suspension et de la turbidité de l'eau, la réduction de la transmission de lumière en profondeur, le * colmatage du fond.

LES MODIFICATIONS DES COMMUNAUTES VIVANTES résultant des altérations précédentes sont : la réduction des densités numériques de Diatomées et de Macroinvertébrés benthiques (la diversité spécifique est peu affectée), une réduction de la teneur du Périphyton en pigments photosynthétiques, un abaissement de la production primaire, une réduction de la biomasse des Macroinverté- 
brés: un ralentissement de la colonisation du substrat par cette faune, une augmentation de la mortalité des cufs et alevins de Truites, incubés sous graviers (* colmatage * par les limons déposés), un ralentissement des éclosions des cufs de Truites, une réduction des effectifs et de la biomasse des populations ichtyaires (prédatrices des Macroinvertébrés).

LES MOYENS DE LUTTE contre ce type de nuisances résident dans l'application de la Législation, notamment des nouveaux textes concernant les Carrières. La principale mesure technique pouvant supprimer la pollution mécanique, due aux effluents de lavage des granulats, consiste à laver les matériaux extraits en circuit fermé (recyclage de l'eau).

II convient également d'interdire les extractions dans le lit mineur des cours d'eau. Quant aux aménagements hydrauliques, il est indispensable que leur conception tienne compte, non seulement de critères hydrauliques, mais aussi des impératifs hydrobiologiques.

\section{SUMMARY}

\section{ECOLOGICAL INCIDENCES OF DREDGING UP ALLUVIAL MATERIALS AND REGULATION OF STREAMS ON THE AQUATIC ECOSYSTEM.}

Granule exploitations (dredging in rivers, and/or badly decanted sewage from sand and gravel washing operations) and some hydraulic works (straightening. gauye modifications), profoundly disturb the aquatic ecosystem

We tried, from 1974 to 1978 , to bring into light these nuisances in three streams in MASSIF CENTRAL : the LOIRE, ALLIER, DORE rivers.

BIOTOP MODIFICATIONS essentially are : the standardization and the unstabilization of the bed, an increase in the rate of suspended particles and water turbidity with reduction of light transmission and bottom clogging.

MODIFICATIONS OF LIVING COMMUNITIES, resulting from deteriorations mentioned above, are : a reduction of Diatoms and benthic invertebrate populations (species diversity is little affected), a reduction of photosynthetic pigments, a decrease in primary production, a reduction of Macro-invertebrate biomass, a slackening of substratum's colonization by this fauna, an increase of Trouts'eggs and fry mortality (spawning-bed warping by deposed slime), a slackening of Trouts' eggs hatching, a reduction of numbers and biomass of fish populations (Macroinvertebrate consumers).

MEANS OF STRUGGLE against these nuisances are : the application of Legislation, especially of the new laws concerning Quarries.

The main technic step to suppress a mecanic pollution», due to the effluents of washing operations, consists in washing materials extracted in closed circuit (water recycling).

It is a!so necessary to prohibit dredging in the bed of rivers. As for hydraulic works, their pre!iminary studies have to take into account, not only hydraulic criterions, but also hydrobiological requirements.

\section{CIRCONSTANCES ET OBJET DE L'ETUDE}

Le présent travail a été effectué en plusieurs étapes, sur la Loire, I'Allier et la Dore, d'août 1974 à juillet 1978 (Fig. 1) : 


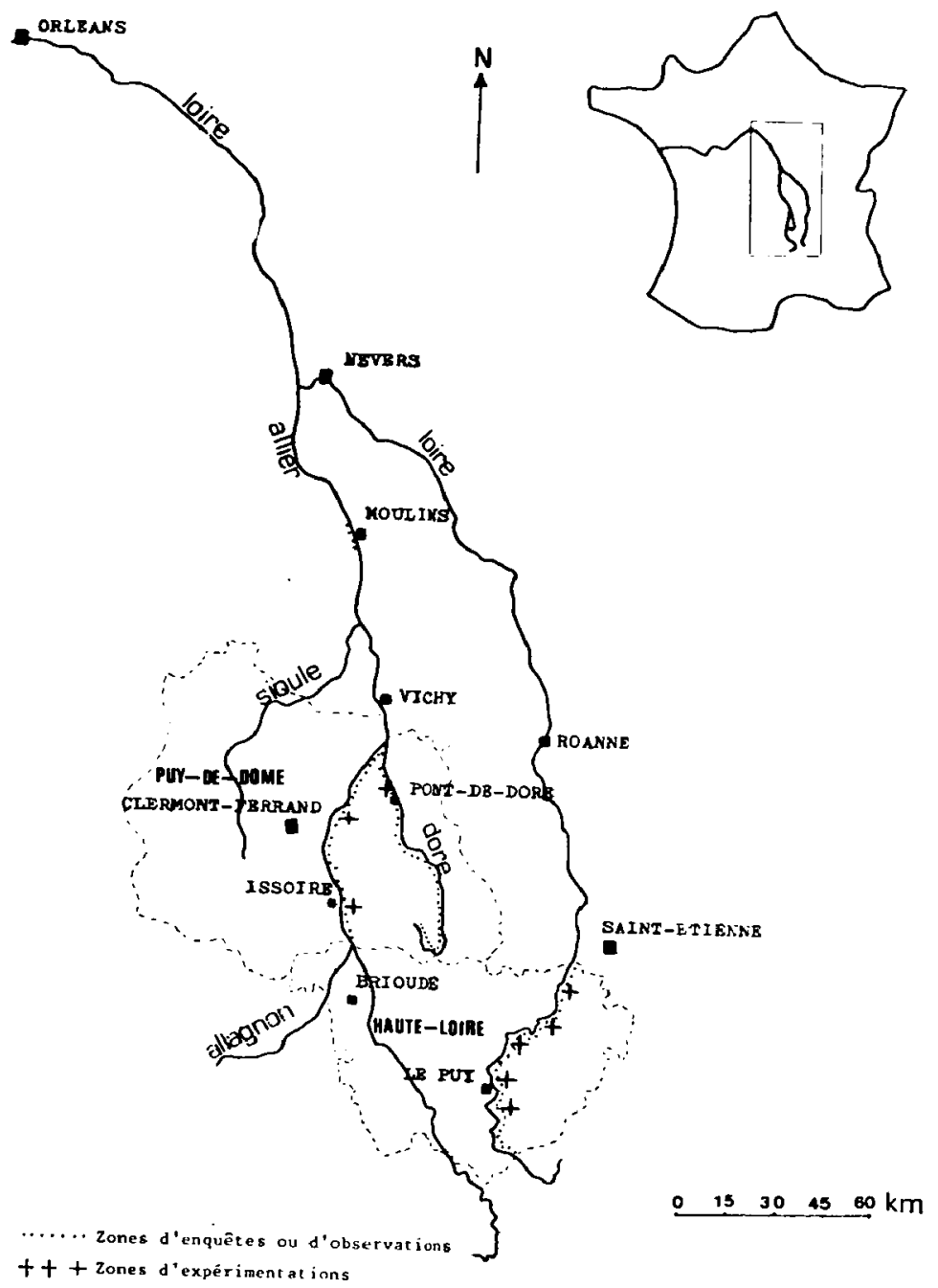

Fig. 1 : Situation géographique de l'étude.

- A la 6e Délégation Régionale du Conseil Supérieur de la Pêche, de 1974 à 1976, par P. CLAVEL, Y. HAMON, C. ROMANEIX.

- Au Laboratoire de Zoologie et de Protistologie de I'Université de Clermont II. en 1977-78, par B. BOUCHAUD et P. CLAVEL.

II concerne les incidences des extractions de granu'ats d'alluvions et de l'aménagement des cours d'eau sur l'écosystème aquatique, et a permis d'établir un constat, d'envisager les textes législatifs et rég!ementaires s'appliquant à ce type d'exploitations, et de proposer des mesures de lutte contre les nuisances occasionnées.

Les perturbations observées et analysèes, dues aux extractions dans le lit mineur et au rejet des eaux de lavage de matériaux, affectent

- le BIOTOPE, dans ses caractéristiques morphodynamiques, dans la physicochimie de l'eau, la transmission de l'énergie lumineuse et la sédimentation. 
- Les COMMUNAUTES VIVANTES (Biocénoses) constituant l'édifice trophique producteurs primaires (périphyton, végétaux supérieurs); consommateurs primaires et secondaires (macroinvertébrés benthiques, poissons).

\section{A. LES MODIFICATIONS DU BIOTOPE.}

\section{1 - Morphodynamique du lit.}

Les rectifications et recalibrages (secteur du pont autoroutier B 71, sur la Dore et sur l'Allier) entrainent l'uniformisation et la déstabilisation du milieu (Fig. 2 et 2 bis).

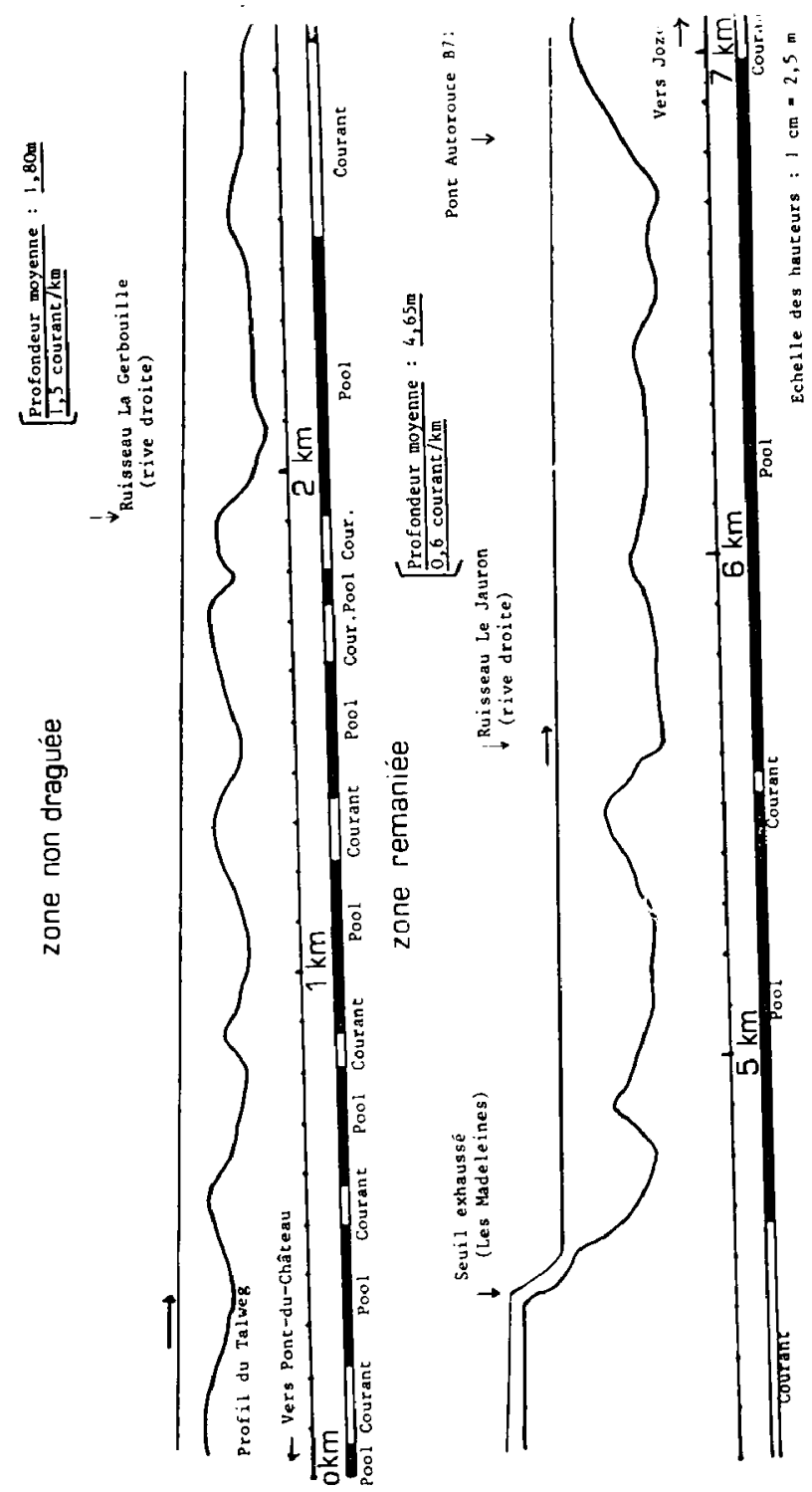

Fig. 2 : Profils en long, sur l'Allier, à l'aval de Clermont-Ferrand, dans le secteur témoin et dans le secteur dragué (rectification du cours d'eau). 
Fig. 2b : La Rivière DORE dans le secteur étudié (en février 1978).

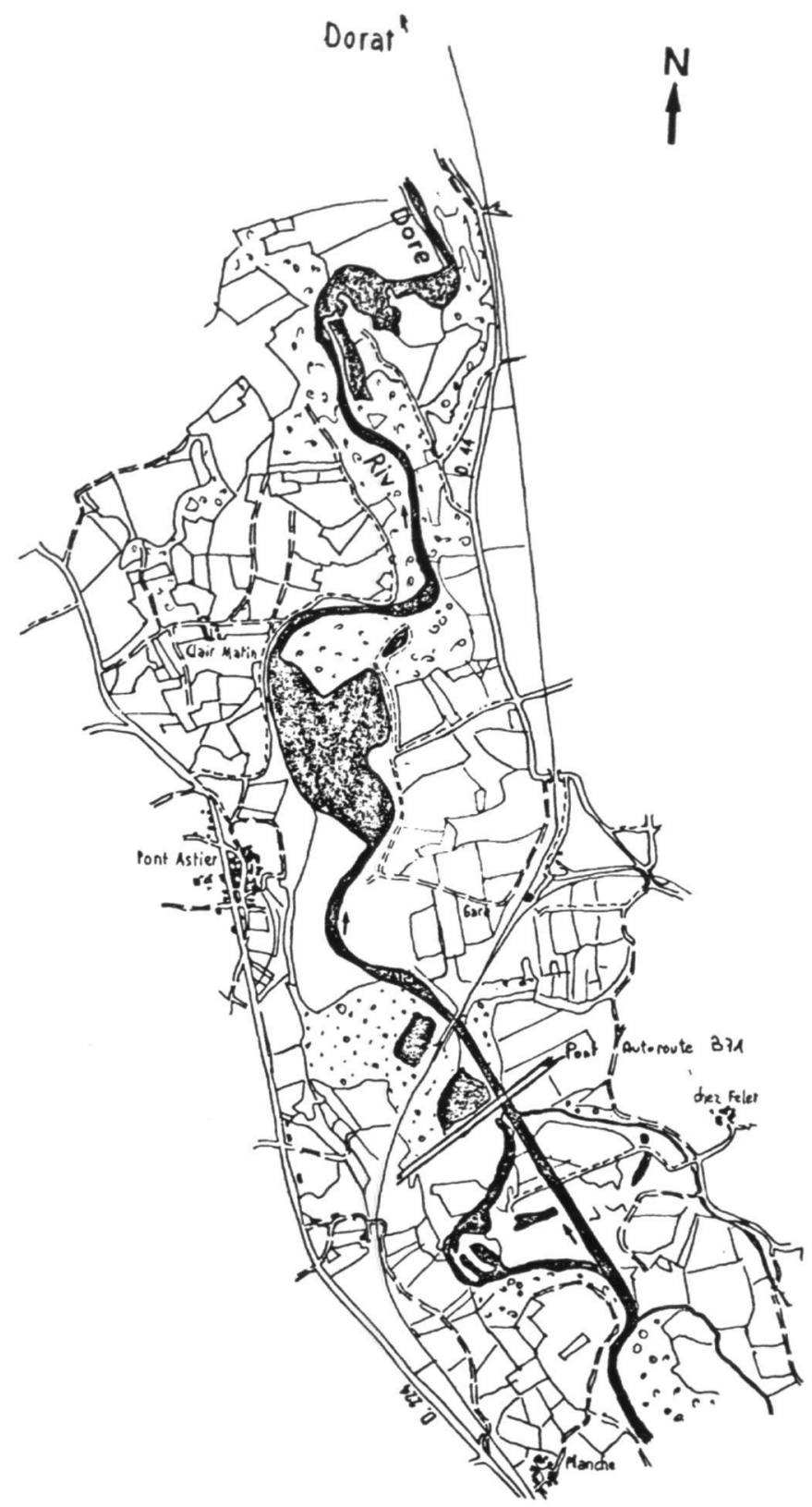

Daprès $*(C)$ carte I.G.N. * 1/25000 feuille Thiers $N^{*} 3-4$ 
- Erosion régressive en amont des dragages, se manifestant par une instabilité du substrat (stations 1 et 6 sur la Dore) ou un exhaussement de seuil (Les Madeleines, le pont de Régemortes, sur l'Allier).

- Constitution de plans d'eau faisant office de pièges temporaires à sédiments (Pont-Astier sur la Dore, aval des Madeleines sur l'Allier, Coubon sur la Loire) et modifiant le niveau typologique théorique de la rivière (* cyprinisation - accélérèe)

- Engravement du lit (apport de matériaux entrainés par l'accélération du courant) à l'aval des zones rectifiées : aval seuil de la station $O$, sur la Dore. Phénomènes de divagation à l'aval des * plans d'eau *, érosions de berges (station 2 sur la Dore).

- Instabilité des berges dans les secteurs remaniés (station O. Dore; Secteur rectifié à l'aval des Madeleines, sur l'Allier).

- Risques encourus par les nappes phréatiques (abaissement de leur niveau, perturbation de leur écoulement, exposition des aquifères aux pollutions de surface).

\section{2 - Physico-chimie de l'eau.}

La plupart des composantes physico-chimiques de l'eau ne sont pas affectées par les extractions et les rejets d'eau de lavage. Seuls, les taux de Matières en Suspension (MES) et la turbidité de l'eau sont augmentés à l'aval.

Les effluents mal décantés ont une charge variant de 400 à $35000 \mathrm{mg}$ (poids sec) de MES/litre. La charge moyenne de celui de la gravière MISSON est de $4400 \mathrm{mg} / \mathrm{l}$ ce qui représente un déversement journalier dans la DORE, de 28 tonnes de MES.

Le dragage dans le lit mineur remet également en circulation un tonnage considérable de *fines". L'augmentation de la turbidité et des MES est particulièrement importante en période d'étiage. Elle est encore sensible près de $5 \mathrm{~km}$ à l'aval de l'effluent, sur la Dore.

\section{3 - Transmission de l'énergie lumineuse.}

Une turbidité élevée de l'eau réduit la quantité de lumière pénétrant en profondeur. (Parallèlisme entre l'augmentation des MES et les diminutions de luminosité). Les radiations bleues sont les plus affectées. A l'étiage ou pour des débits moyens, cette réduction est encore nette $3 \mathrm{~km}$ à l'aval du rejet (Fig. 3). Le coefficient d'absorption lumineuse a une valeur moyenne de 2,22 à la station témoin, de 3,45 une centaine de mètres après le débouché de l'effluent, et de 2,84 un kilomètre et demi à l'aval.

\section{4 - Sédimentation des M.E.S.}

Les MES provenant des dragages ou des rejets d'eau de lavage, outre la turbidité et la réduction de la transmission lumineuse qu'elles provoquent, sédimentent progressivement vers l'aval. Ces "fines", composées essentiellement de particules inférieures à 200 microns, tendent à colmater les interstices entre les cailloux et graviers, et les végétaux immergés Leur sédimentation sur substrats artificiels en faciès benthique représente un poids 3 fois plus élevé $(500 \mathrm{~m}$ à l'aval d'une gravière sur la Loire) qu'à la station témoin amont, et 2 fois $1 / 2$ plus 
fig 3 : Pénétration de l'énergie lumineuse

Sortie du 8 juin 1977

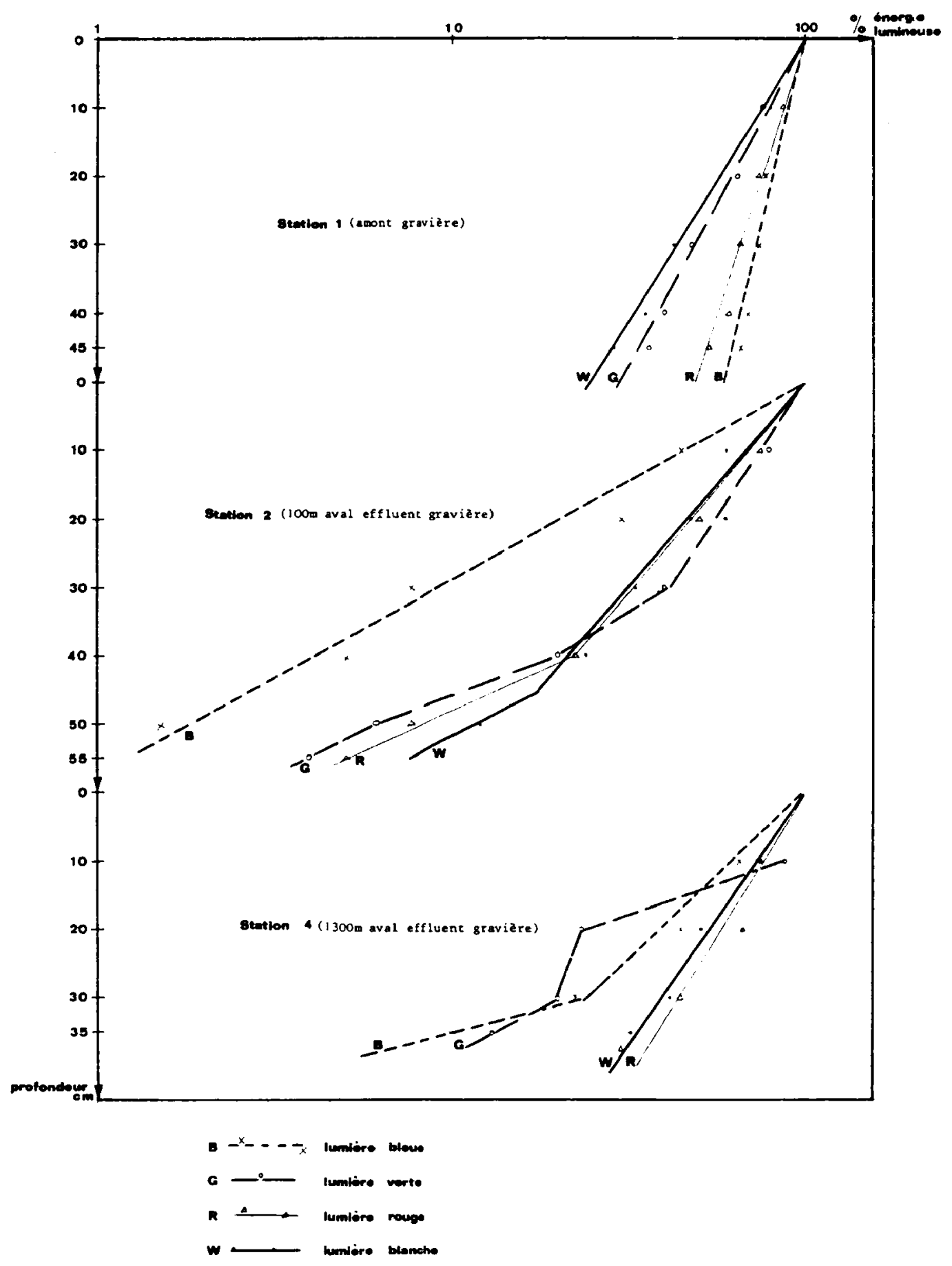


élevè, $1200 \mathrm{~m}$ à l'aval. Sur la Dore, en faciès lotique, l'augmentation est plus faible : poids multiplié par 2, $100 \mathrm{~m}$ à l'aval du rejet, par 1,3,600m à l'aval.

\section{LES MODIFICATIONS DES BIOCENOSES.}

\section{1 - Le périphyton.}

- La diversité spécifique des communautès de Diatomées (150 taxons dénombrés) n'est pratiquement pas appauvrie à l'aval de la gravière MiSSON. Seu'es, les densités numériques sont réduites, particulièrement durant l'ètiage, où nous enregistrons des diminutions (par rapport à la station témoin) allant de 54 à $94 \%, 3 \mathrm{~km}$ à l'aval du rejet.

Les divers indices biotiques calculés ne permettent pas de caractériser la pollution mécanique au niveau de ces populations algales.

- La mesure des concentrations de pigments photosynthétiques constitue, par contre, un autre moyen d'évaluer l'impact des MES et de la turbidité sur le périphyton (Fig. 4).

Les teneurs en chlorophylle a (pigment le plus abondant, et absorbant dans le b'eu) sont encore róduites de 49 à $90 \%, 2600 \mathrm{~m}$ à l'aval de l'effluent, en période de débits élevés. A l'étiage, les quantités de pigments sont les plus fortes: la diminution de la chlorophylle a, par rapport au témoin 'amont, atteint 56 à $74 \%$, $2600 \mathrm{~m}$ à l'aval. Il en va de mème pour les teneurs en caroténoïdes.

- Les valeurs extrémes de la production primaire du périphyton sont proportionnelles aux concentrations de pigments correspondantes. Le minimum est obtenu $100 \mathrm{~m}$ à l'aval du rejet. Pendant les basses eaux de l'automne 1977. la réduction de l'assimilaiion photosynthétique est encore observée près de $3 \mathrm{~km}$ à laval (diminution par rapport au témoin, de $75 \%$ sous la surface de l'eau, de $27 \%$ à $40 \mathrm{~cm}$ de profondeur).

\section{2 - Les macrophytes.}

La production primaire des Renoncules et Callitriches est relativement faibie sur l'ensemb'e du secteur étudié. Les incidences négatives de la réduction de luminosité sur leur assimilation chlorophyllienne ont été relevées lors de deux cxpérimentations, $100 \mathrm{~m}$ en aval de l'effluent, pour les Callitriches, jusqu'à $600 \mathrm{~m}$ aval, pour les Renoncules. Des expériences complémentaires seraient nécessaires pour préciser l'ampleur de ces perturbations sur les Végétaux Supérieurs.

\section{3 - Les macroinvertébrès benthiques.}

Les résu!tats obtenus sur la Loire et l'Allier, en 1974, 75 et 76, montrent que les effectifs de ces communautés sont réduits en moyenne de 20 à $60 \%$ sur une distance de $1300 \mathrm{~m}$ à l'aval de la gravière, en faciès lotique; de 27 à $61 \%$ sur la même distance, en faciès lentique.

La diminution des biomasses est particulièrement nette sur $1300 \mathrm{~m}$, pendant la période de production maximale (avril sur la Loire)

- 78 à - $81 \%$ en lotique (90\% de la surface en eau), soit $121 \mathrm{~kg} / \mathrm{ha}$, $1300 \mathrm{~m}$ à l'aval de l'exploitation contre $658 \mathrm{~kg} / \mathrm{ha}$, dans le secteur témoin (Fig. 5). 


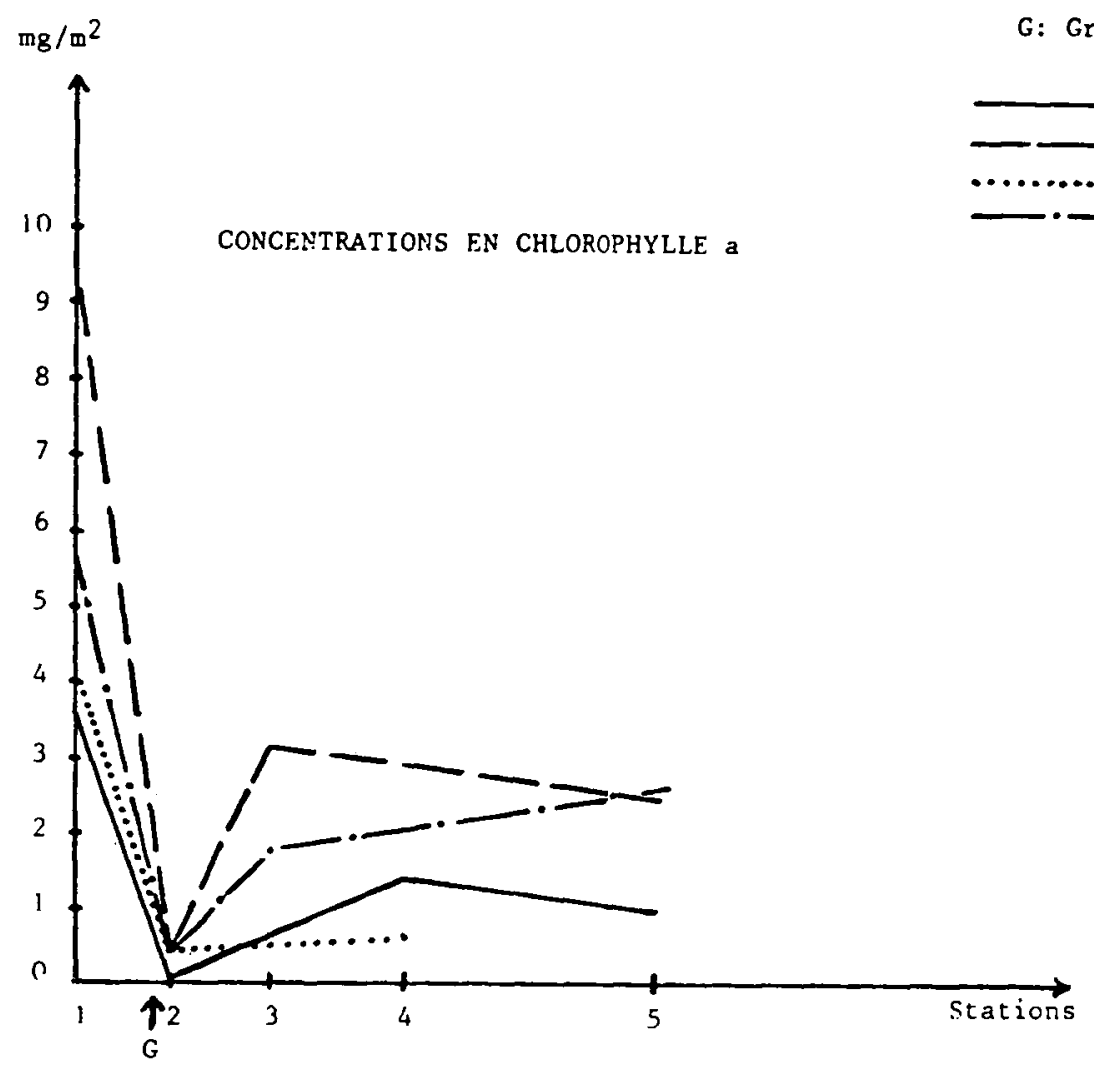
$05 / 10 / 77$

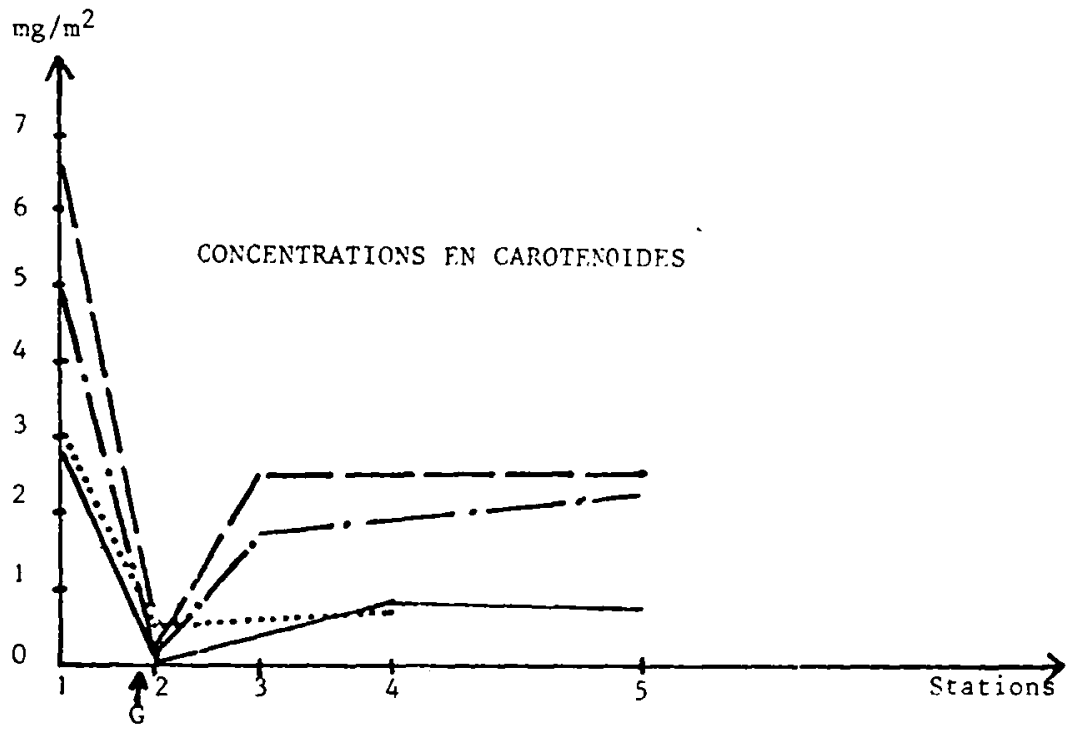

Fig. 4 : Variations des teneurs en chlorophylle a et caroténoïdes lors de quatre prèlèvements (28 jours d'immersion des substrats artificiels). 


\section{faciès lotique $\mid 80 \%$ de la surface des stations $\mid$}

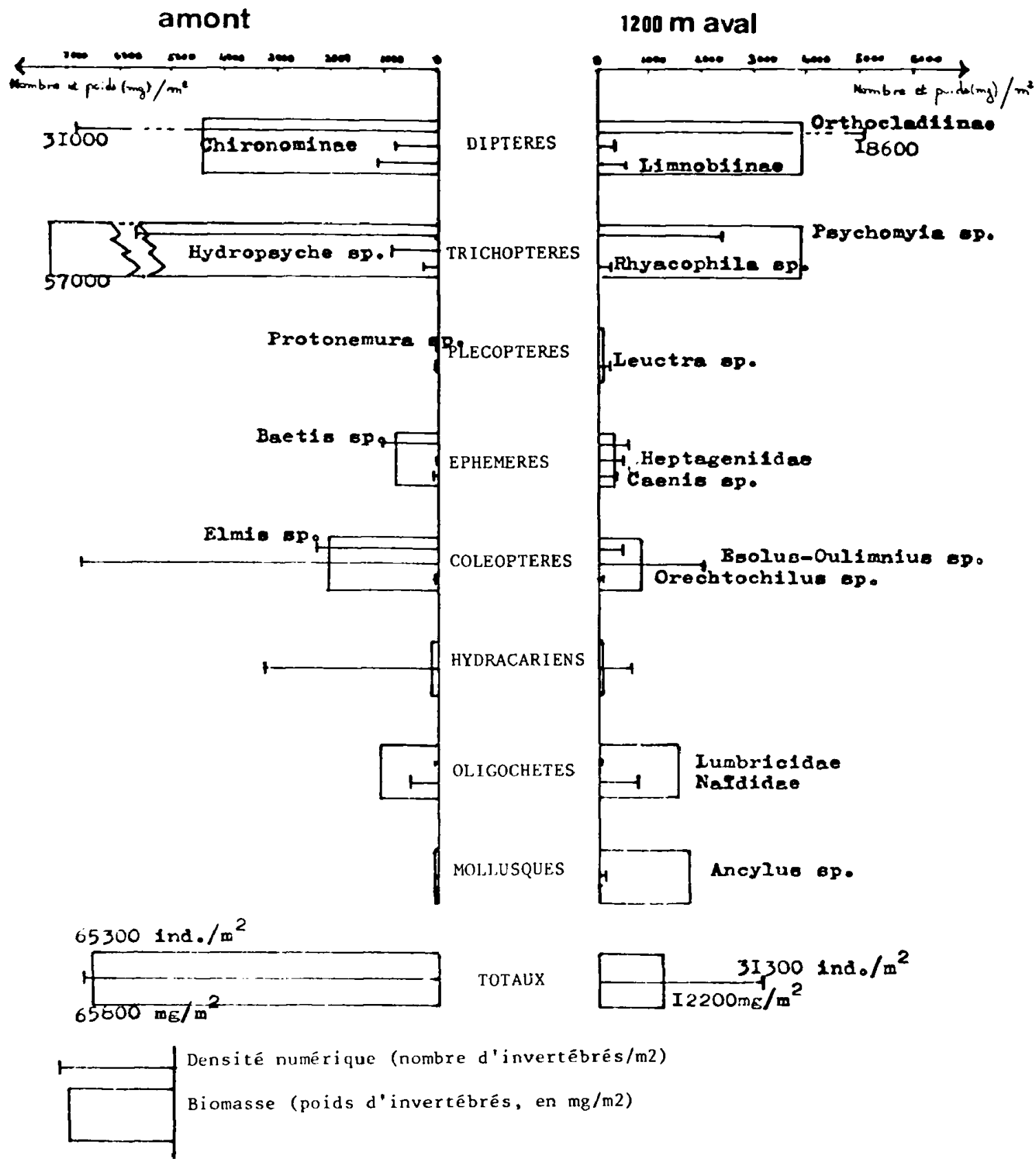

Fig. 5 : Densités et biomasses d'invertébrès benthiques, à l'amont et à l'aval (1200 m) d'une gravière, sur la Loire en avril 1976. 
Cet appauvrissement des communautes affecte pratiquement tous les groupes faunistiques, principalement les Diptères. Trichoptères, Coléoptères et Hydracariens.

Le colmatage du substrat par les "fines" provoque un ralentissement de sa colonisation par les macro-invertébrés (expérimentation sur substrats artificie!s).

Un deuxième phénomène semble mis en évidence sur la DORE, en 1977 : l'instabilité du lit à l'amont d'une zone draguée (surdimensionnée), entraine une réduction des effectifs et des biomasses de macroinvertébrés.

La méthode des Indices Biotiques, quant à elle, ne permet pas de deceler une pollution mécanique.

\section{4 - Les poissons.}

- L'étude du régime alimentaire des Barbeaux (Barbus barbus L.) et Vandoises (Leuciscus leuciscus L.), en amont et $800 \mathrm{~m}$ en aval d'une gravière, sur la LOIRE, nous a montré que les groupes DIPTERES + TRICHOPTERES + EPHEMERES (représentant plus de $85 \%$ de la biomasse tota'e du secteur de rivière) constituaient $99 \%$ du contenu du tube digestif de ces poissons (Fig. 5b). Or. Diptères et Trichoptères voient leurs biomasses particulièrement réduites à l'aval do l'exploitation (Cf. plus haut).

- L'expérimentation réalisée sur l'incubation d'ceufs de Truites (Salmo trutta fario L.) en amont, 150 et $750 \mathrm{~m}$ aval d'un effluent de gravière sur l'Allier, nous a également révélé que le colmatage du lit par les "fines" déversées, provoquait sur les frayères de Salmonidés des taux de mortalité très élevés (de 99 et $40 \%$ pour les œufs; de 76 et $23 \%$ pour les alevins). Par ailleurs, le dépôt des MES entraine un ralentissement des éclosions (Fig. 6).

- Un inventaire pisciaire (pêches électriques) effectué sur la Loire (mêmes secteurs que ceux de l'étude alimentaire), nous a indiqué une diminution de $28 \%$ des effectifs et de $17 \%$ des biomasses à l'aval de la gravière (par rapport à la section témoin).

L'ensemble des espèces est affecté, notamment les Ablettes (- $99 \%$ ), les Vandoises (-93\%), les Vairons $(-68 \%$ ) et les Goujons (- $48 \%$ ), à l'exception des Barbeaux $(+124 \%$ ) et des Chevesnes $(+31 \%)$ qui "profitent " de la réduction des populations des autres espèces. La structure de la communauté cst modifiée : 3 espèces constituant $83 \%$ de l'effectif aval contre 5 espèces à l'amont. (Fig. 7).

Cette perturbation d'un peuplement ichtyaire (dernier niveau de l'édifice trophique) peut s'expliquer

- par les réductions des communautés vivantes des divers niveaux inférieurs : producteurs primaires (a!gues) et consommateurs primaires et secondaires (macroinvertébrés benthiques):

- par l'action directe des "fines " colmatant les frayeres;

- oar les modifications morphodynamiques du cours d'eau résultant des dragages : uniformisation et déstabilisation du lit impliquant la suppression des abris et de la végétation fixée (susport pour les œufs de ceriaines espèces, et habitat de macroinvertébrés), la rupture de l'alternance pools (zones de repos) et courants (zones d'alimentation), et, la constitution d'obstacles aux migrations (exhaussements de seuils) (Fig. 8). 


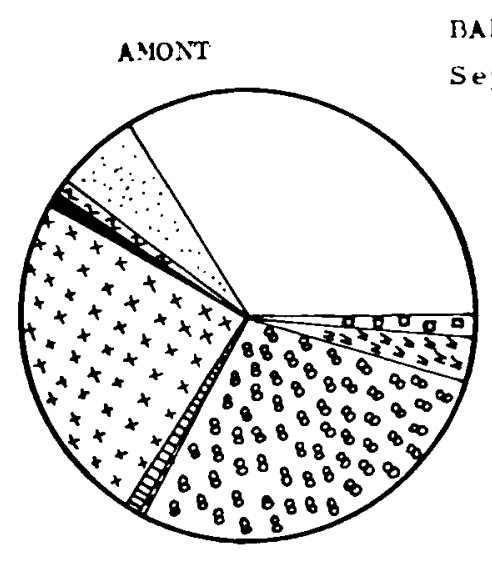

BARBLALX

AlAL

eptembre 1975

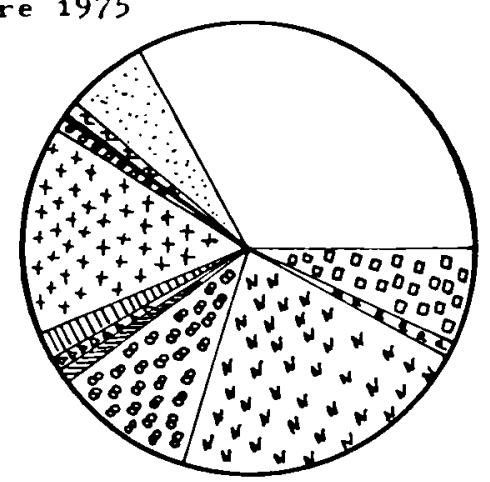

]$^{\text {Orthocladiinae }}$

Chironominae

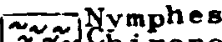

2 $\sim^{2} C_{h}$ ironomidae

BARBEAUX

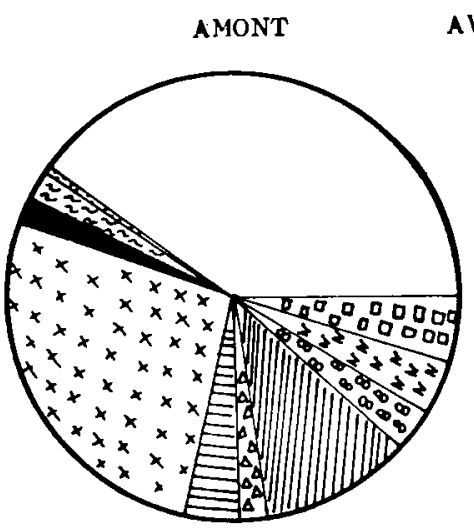

AVAL

Limnobiinae

$60^{\circ} 0^{S}$ imuli dae

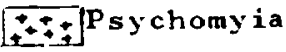

Hydropsyche

AAARyacophila

Ylyol igoplect rum

8.80etis

$\widetilde{N_{N}}$ Nématodes

Prosites

a 0 a d ivers

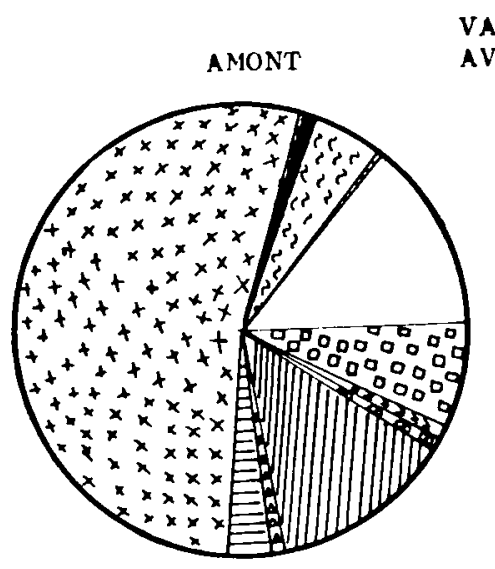

VANDOISES

AVRIL 1976 AVAL

Fig. 5b : Composition moyenne des contenus stomacaux de poissons capturés à l'amont et $800 \mathrm{~m}$ à l'aval d'une gravière, sur la Loire, à Coubon (43)

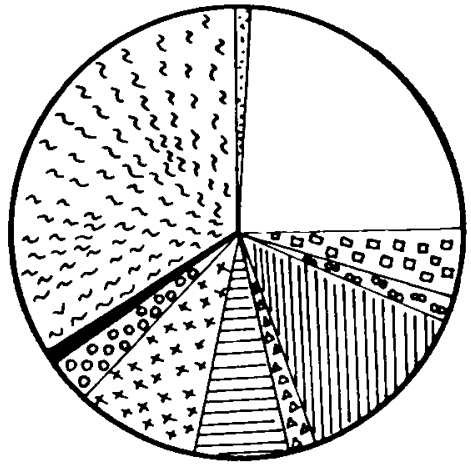



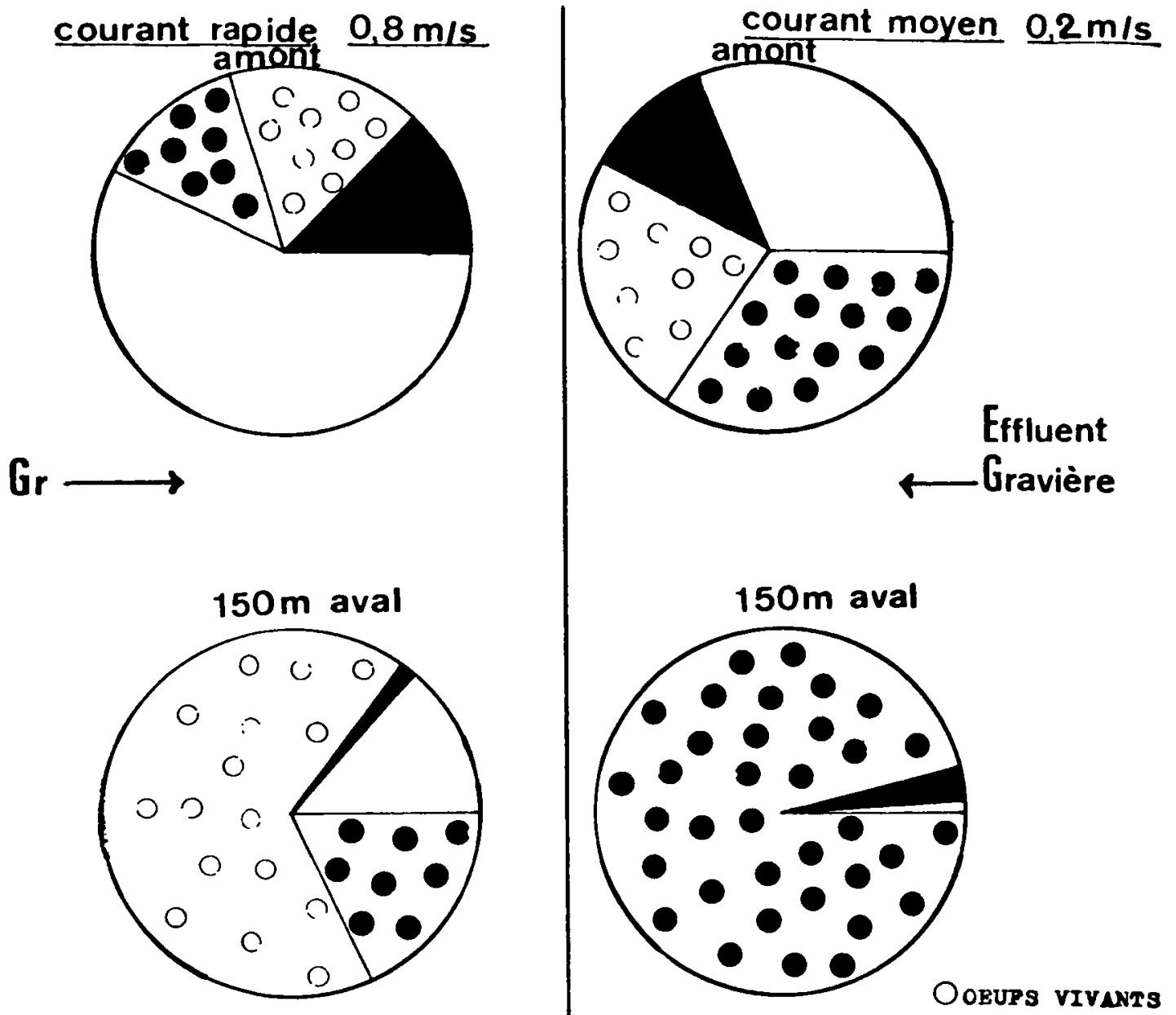

OOEUPS vivants
OEUPS MORTS
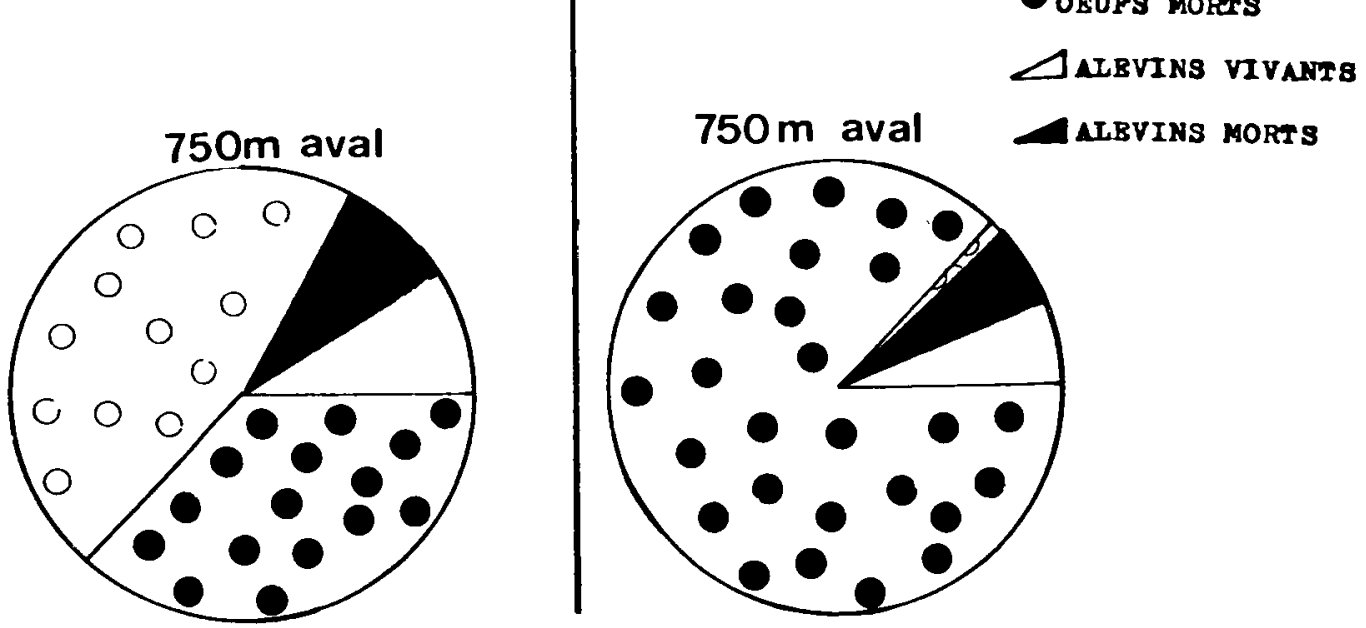

Fig. 6 : Influence du colmatage des graviers par les MES sur le développement des œufs et alevins de truites, après 20 jours d'exposition, le 1 er mars 1976 (Rivière ALLIER, près d'ISSOIRE). 


\section{DENSITÉ NUMÉRIQUE}

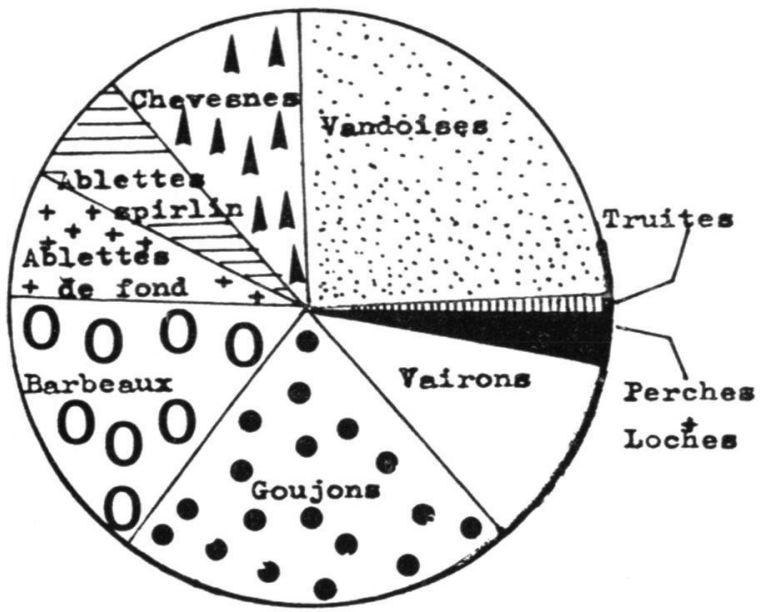

a mont

2865 ind / ha

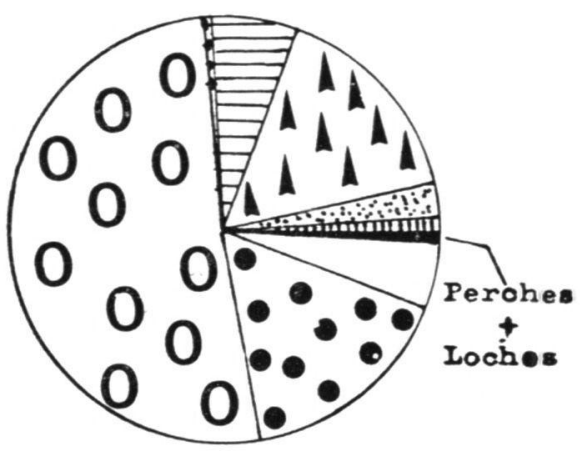

aval

2064 ind / ka

\section{BIOMASSE}
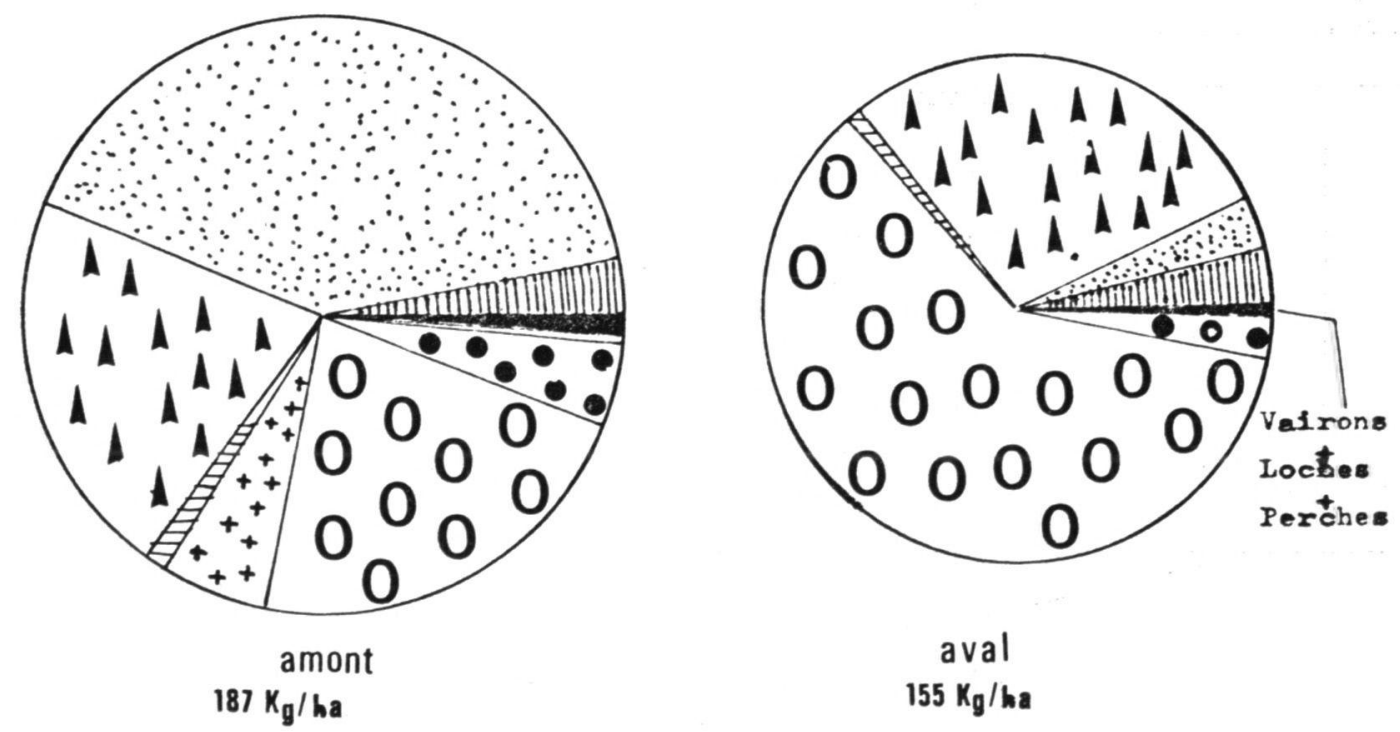

Fig. 7 : Peuplement ichtyaire estimé à l'amont et à l'aval d'une gravière sur la Loire (septembre 1975). 


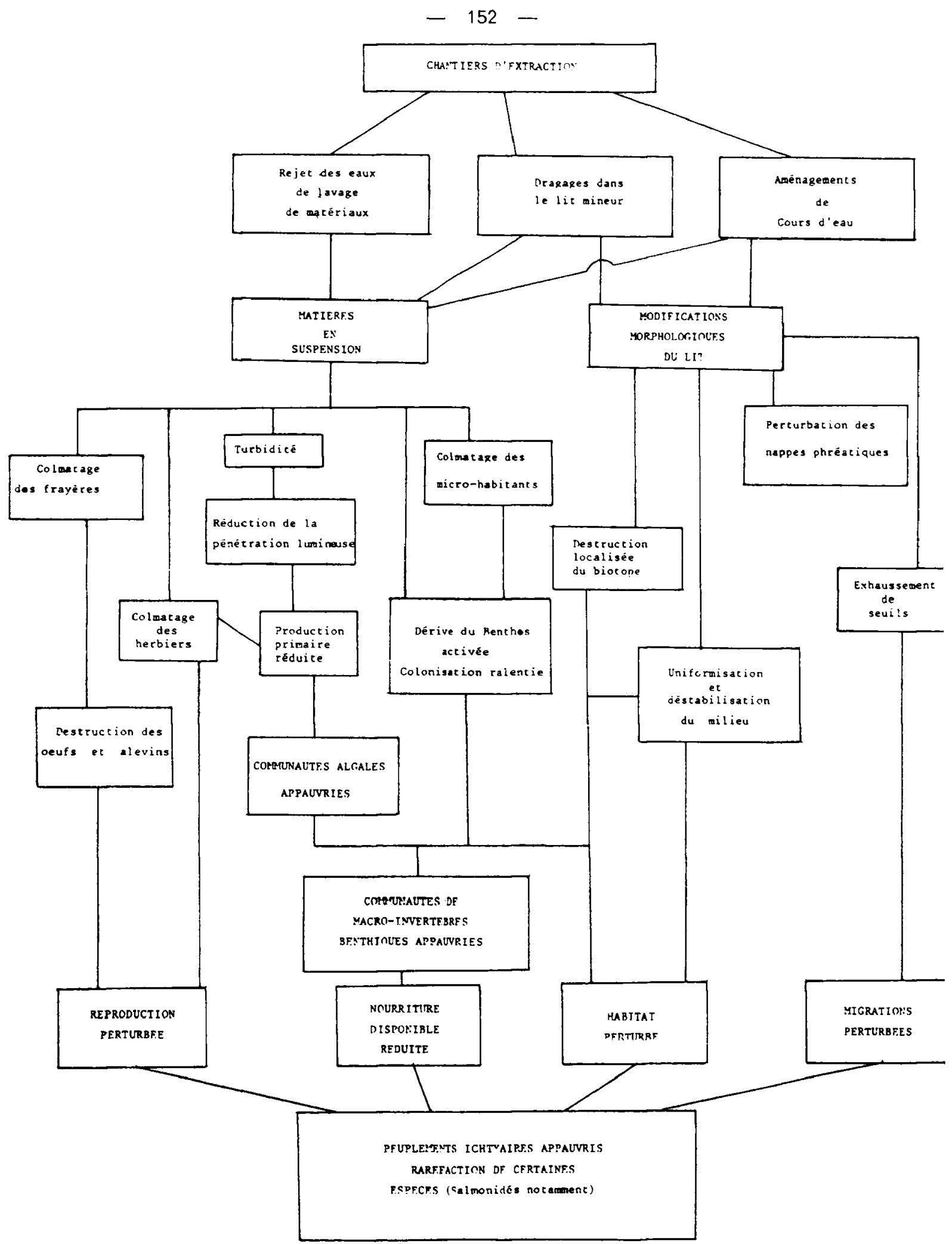

Fig. 8 : Schéma de l'impact des chantiers d'extraction sur l'écosystème aquatique. 


\section{LES MOYENS DE LUTTE CONTRE CE TYPE DE NUISANCES.}

\section{1 - Aspect juridique et Taxations.}

- Les textes législatifs en vigueur, et la nouvelle réglementation s'appliquant aux gravières (Loi sur la Protection de la Nature et décret sur les études d'impact; Loi sur les installations classeees; Réforme du Code Minier), renforcent le pouvoir et les responsabilités du Préfet et des administrations concernées (DDA, DDE, Service de I'Industrie et des Mines). Ils prescrivent la prise en compte de l'environnement dans tout projet ou réalisation susceptibles de lui porter atteinte, et les mesures destinées à éviter ou à réduire ces perturbations.

L'arsenal juridique parait donc suffisant. La protection du milieu aquatique dépend de son application.

Au plan départemental, nous demandons que dans les Arrêtés préfectoraux, les mesures à prendre pour limiter ou supprimer les pollutions mécaniques, soient explicitement indiquées aux exploitants. II nous parait, dans ce sens, indispensable que les extractions dans le lit mineur d'un cours d'eau, ne soient plus autorisées.

Un facteur ambigü est aussi constitué par le fait que les Directions Départementales de l'Equipement interviennent, à la fois dans les autorisations d'ouverture des exploitations et leur contrôle, et comme client, direct ou indirect, de ces entreprises qui leur fournissent les matériaux de viabilité.

- La taxation des Agences Financières de Bassin concerne les prèlèvements d'eau et les rejets. Cette "imposition " semble dissuasive sur ce dernier point, mais toutes les exploitations ne sont pas recensées et les contrôles du bon fonctionnement des bassins de décantation sont rares.

Par contre, il n'existe pas de taxation de l'operation de dragage dans le lit mineur, alors que ce type d'extraction est très préjudiciable au milieu aquatique, comme nous l'avons montré.

\section{2 - Mesures techniques.}

\section{a) Les exploitations de granulats.}

- En nous appuyant sur l'étude du C.E.T.E. de Lyon*. nous demandons la généralisation du lavage des matériaux en circuit fermé comme solution "parfaite" au problème des rejets d'eau mal décantée. Ce système réduit, en outre, considérab!ement les taxations des exploitants par l'Agence de Bassin.

- Une question reste posee : le devenir des boues retirées des bassins de décantation. Une étude approfondie dans ce domaine semble nécessaire. Parfois recyclées partiellement (récupération de sable), utilisées en agriculture ou mises à la décharge publique, elles servent aussi à combler d'anciennes excavations du lit majeur..

- Ceci nous amène à évoquer brièvement le réaménagement des carrières. Ne serait-il pas possible de limiter, dans certains cas, la profondeur des extractions sur les terrasses alluviales, de façon à ne pas atteindre le niveau de la nappe phréatique (réduisant ainsi les perturbations de l'aquifère), et à susciter une remise en état à des fins agricoles.

\footnotetext{
Centre d'Etudes Techniques de l'Equipement, Laboratoire Régional de Clermont-Ferrand.
} 
Les aménagements en étangs de pêche ou en bases de loisirs, bien qu'ètant souhaitables, sont, dans certaines régions, les seuis exemples de realisations.

Il conviendrait également de promouvoir la création de réserves ornithologiques, pour les Limicoles notamment, dans des ballastieres de grande superficie, partiellement noyées (bords de Dore et d'Allier...).

- Des recherches sont aussi à entreprendre dans lutilisation do matériaux de substitution là oủ les granulats d'alluvions ne sont pas indispensables. Pour les remblais de routes (ou d'autres travaux), les residus d'incinération des ordures ménagères, matériaux de démolition du bâtiment et des travaux publics, déchets ménagers divers (verre plastique), schistes houiller, stériles de mines et laitiers de hauts-fournaux, peuvent trouver là un bon exutoire.

- Les alluvions constituant un réservoir et un "massif filtrant "pour les eaux destinées à la consommation humaine, il est essentiel que cet objectif soit maintenu premier dans le cadre d'une gestion des réserves alluviales. L'article 109-1 du Code Minier et les créations de ZERC (Zones d'exploitation et de réaménagement coordonnés), prévues dans le futur décret d'application, spécifique aux carrières, sont appelés à jouer un rôle primordial dans ce domaine.

\section{b) Les aménagements de cours d'eau.}

De telles réalisations - lorsquielles apparaissent nécessaires pour préserver zones urbaines, agricoles, routes, ouvrages d'art, etc... menaces par les inondations, doivent être conçues non seulement selon des critères hydrauliques, mais prendre aussi en compte les impératifs hydrobiologiques

Du respect de ces deux conditions, au niveau de l'ensemble d'un bassin hydrographique, dépendent une gestion efficace et positive, et une protection des cours d'eau, compatible avec les divers intèrêts en prèsence

- alimentation en eau potable et protection des nappes aquiferes;

- protection des habitats et des terres agricoles contre les inondations;

- protection des ouvrages d'art;

- tourisme et loisirs liés à l'eau (pêche, baignade, etc...);

- extraction de matériaux.

Divers exemples ont montré que les opérations de rectification et de recalibrage, effectuées pour évacuer les crues, rapidement et sans débordements (et fournissant, en même temps, des matériaux prélavés pour les Travaux Publics), se révèlent nuisibles non seulement pour l'écosystème aquatique, mais aussi à plus ou moins lang terme, pour les zones "amérıagées" elles-mêmes (engravement progressif du lit provoquant de nouvelles divagations du cours d'eau et nécessitant donc de nouveaux dragages) et pour les zones aval (fortement urbanisées) qui reçoivent, de ce fait, des crues plus violentes.

Un cours d'eau ne saurait constituer, en aucun cas, une réserve de matériaux à extraire, ou un canal uniforme assurant la circulation rapide d'un élément mobile, l'eau. II s'agit d'un milieu vivant diversifié, en équilibre dynamique ; caractères dont protection et gestion doivent tenir compte, dans le cadre d'une politique cohérente de Bassin, en concertation avec les différents utilisateurs: consommateurs d'eau, que nous sommes tous, pour l'alimentation et les loisirs, aménageurs, industriels et exploitants de granulats, agriculteurs et pêcheurs. 


\section{BIBLIOGRAPHIE SOMMAIRE}

ANNALES DES MINES (1976). L'approvisionnement en produits de carrieres, no $12, p .13-178$.

ARRIGNON J., ROUYER G., CHANCEREL F. (1974). La sauvegarde des intérêts piscicoles au cours des travaux d'aménagement des cours d'eau. Compte rendu session de la SHF. La Houille Blanche, no spécial. 2/3 - 1975. p. 117125.

BOU C. (1977). Conséquences écologiques de l'extraction des alluvions récentes dans le cours moyen du Tarn. Bull. Ecol., t.8, 4, p. 435-444.

BOUCHAUD B., CLAVEL P. (1978). Les exploitations de granulats d'alluvions et les amènagements de cours d'eau dans l'écosystème aquatique. Leurs incidences sur le périphyton et la production primaire dans le cours inférieur de la Dore. Bilan 1974-1978 sur Loire, Allier, Dore. Rapport Labo. Zoo. Hydrob. rég. Université Clermont II, $213 \mathrm{p}$.

BURLAT M.F. (1975). Les ballastières dans la vallée de la Risle. Bulletin d'information du CSP, 101, p. 20-33.

CENTRE D'ETUDES TECHNIQUES DE L'EQUIPEMENT (1977). Optimisation des méthodes de lavage et de décantation dans les exploitations de roches massives et de matériaux alluvionnaires. Phases 1 et 2, p. 1-75

CLAVEL P.. HAMON Y., ROMANEIX C. (1977). Effets des extractions de matériaux alfuvionnaires sur l'environnement aquatique, dans les cours superieurs de la Loire et de l'Allier. Thèse 3 e cycle. UER. Sciences, Clermont-Fd, et ingéniorat ESITPA, Le Vaudreuil, $186 \mathrm{p}$

CLAVEL P., CUINAT R.. HAMON Y., ROMANEIX C. (1978). Effets des extractions de materiaux alluvionnaires sur l'environnement aquatique. Synthèse des résultats de 1974 à 1976. Conseil Supérieur de la Pêche, 6e DR, Bulletin français de pisciculture, 268, p. 122-154.

COMITE DE GESTION DE LA TAXE PARAFISCALE SUR LES GRANULATS (1977). Les carrieres, richesse ou plaie. 3 plaquettes de $12 \mathrm{p}$.

C.T.G.R.E.F. (1976). Effets biologiques et écologiques des extractions de matériaux dans le lit des cours d'eau (Pollution "mécanique"). Rapport préliminaire. Observations réalisées sur le cours inférieur du Doubs no 12, Antony, p. $1-26$

C.T.G.R.E.F. (1978). Effets biologiques et écologiques des extractions de matériaux dans le lit des cours d'eau (Pollution "mécanique "). Rapport no 2. Modification du peuplement ichtyologique du cours inférieur du Doubs D.Q.E.P.P. Antony, p. 1-19.

DE MANDER M. HARMEY J. (1977). Dégradations, nuisances et pollutions occasionnées par l'expioitation des carrières. B.R.G.M., Orléans, p 1.61.

HUET M., TIMMERMANS J.A. (1976). Influence sur les populations de poissons, des amenagements hydrauliques de petits cours d'eau assez rapides. Station de Recherches des Eaux et Forêts. D. no 46, p. 1-27.

PORCHER J.P. (1975). Déséquilibres écologiques liès à l'implantation de ballastières sur des cours deau de lre catégorie piscicole. Communication à I'A.F.L., C.S.P. 1re Région Piscicole. 
RIEUL L. (1976). L'entretien et l'aménagement des cours d'eau. B.T. Service de l'hydraulique. Genie Rural. p. 23-27.

ROBBE D. (1975) Influence des matieres minérales en suspension sur la qualité des eaux de surface. Rapport de recherche no 49. Laboratoire Central des Ponts et Chaussées, $123 \mathrm{p}$.

ROSENBERG D.M., WIENS A.P. (1975). Experimental sediment addition studies on the Harris River, N.W.T., Canada : the effect on macroinvertebrate drift. Verh. Internat. Verein. Limnol., 19, p. 1568 - 1574.

SERVICE REGIONAL DE L'AMENAGEMENT DES EAUX (1974). Etude du bassin de la Dore. Région Auvergne $23 \mathrm{p}$. + annexes.

VERNEAUX J., VERGON J.P., LARINIER M. (1974) Aspects écologiques des travaux d'aménagement des cours d'eau. Orientations et principes généraux Compte rendu Session S.H.F., La Houille Blanche, 2-3, p. 127-132. 